\title{
Therapeutic Vaccination against T-Cell-Defined Tumor Antigens
}

D uring the recent decades the limitations of conventional cancer therapy involving surgery, radio- and chemotherapy became obvious. Strategies that allow to complement conventional therapies are searched for. 0 ne of them is the vaccination against tumor antigens recognized by $T$ lymphocytes. $D$ uring the past 15 years the knowledge on molecular details of T-cell recognition increased almost exponentially and technical advances allowed to identify an ever growing number of T-cell-defined antigens in human tumors of different histology. This caused a revival of therapeutic vaccination and a strongly intensified research activity in this field, that was joined by most of the academic research institutions in oncology and hematology during the recent years.

$T$ cells recognize short amino acid chains, named peptides, that are processed from cellular proteins. Peptides are transported to the endoplasmatic reticulum, where they non-covalently bind to $H L A$ molecules and from where these complexes are transported to the cell surface. Considering the manifold genetic changes occurring in the course of tumor progression, there is a seemingly endless variety of peptides that should appear preferentially or specifically on the surface of tumor cells. D ozens of such antigens have been identified by now. They can be divided into distinct categories according to their origin and to their specificity of expression:

1. MAGE-type or 'C ancer/Testis' antigens, that are specifically expressed in tumors mostly as a result of demethylation of regulatory sequences;

2. differentiation antigens, whose expression is confined to the parental cell type;

3. point-mutated and viral oncoproteins as well as fusion proteins encoded by translocated genes, whose expression contributes to tumorigenesis;

4. highly individual tumor-specific antigens generated by point mutations occurring incidentally during tumorigenesis and tumor progression, that apparently do not contribute to the malignant phenotype;

5. antigens, that are expressed by normal genes, but are generated by the utilization of alternative reading frames or alternative splicing. We now know that human tumor cells carry multiple antigens that in principle can be recognized by the autologous immune system. However, in tumor patients these antigens obviously do not elicit effective responses. Reasons for this are manifold. A variety of mechanisms lead to T-cell anergy or ignorance. They are induced by tumor-associated molecules that are either expressed on the cell surface or secreted into the extracellular environment of tumor cells. $M$ any of the tumor-associated antigens identified so far have to be classified as autoantigens, because they are also expressed normal cells. T-cell responses against such antigens are controlled by central and peripheral tolerance. In addition, tumors are prone to loosing or downregulating the expression of molecules that enable or favor recognition by $T$ cells.

E arly vaccinations with either allogeneic or autologous tumor cells had no reproducible beneficial effects. But at that time the presence of relevant antigens on tumor cells, from which vaccines were pre- pared, was only hypothetical. Now, the identification of T-cell epitopes on human tumors, the increasing understanding of how peptide antigens are processed, transported and presented by $\mathrm{HLA}$ and the identification of mechanisms leading to immune tolerance, ignorance and escape have opened new routes, along which new strategies of specific immunotherapy can be developed with better chances than ever before. A mong these, the concept of therapeutic - as opposed to preventive - immunization against defined tumor-specific or tumor-associated antigens currently prevails. I t relies on the hypothesis that tolerance or ignorance of antigens expressed in a given compartment can be broken by introducing these antigens into a distant site, where professional antigen-presenting cells like dendritic cells are involved. A long this line, pilot vaccination studies were initiated. Patients were preferentially vaccinated with peptides, autologous dendritic cells loaded with peptides and viral constructs In parallel, new paths for the vaccination against the entirety of tumor antigens present on individual tumors were entered, such as the vaccination with gene-modified tumor cells, with peptide-loaded heat shock proteins or R NA isolated from autologous tumor tissue, and with autologous tumor cells fused to dendritic cells. R egressions of metastases were reported for a minority of patients. It is of course almost impossible to conclude from these early observations that regressions were in a strict sense caused by the vaccinations. It has to be kept in mind that in melanoma and renal cancer, for which most studies were designed, spontaneous regressions might occur more frequently than previously believed. In renal cancer the rate of spontaneous regression can be as high as $6.6 \%$, as recently reported. O nly prospective randomized clinical trials will reveal whether patients undergoing therapeutic vaccination will survive better. $\mathrm{H}$ owever, before such time- and energy-consuming studies will be undertaken, one would wish to find out in pilot or phase I/II studies,

- in which patient subgroups,

- against which antigens or antigen categories, and

- with which vaccination strategy

detectable and efficient anti-tumor T-cell responses can be induced. We assume that animal models will not help very much in that respect. In patients mainly those studies will be helpful that provide not only clinical but also immunological parameters with techniques that have been developed and established during the past years. Such parameters include immunological characteristics on the tumor side and both a quantitative and qualitative characterization of specific T-cell responses. These will be essential prerequisites to compare and improve therapeutic vaccination strategies and to select early in time parameters with a high potential to predict a priori success or failure. E specially when considering other promising new concepts of cancer therapy, such as anti-angiogenesis and inhibition of signal transduction, it will be very important to let single strategies mature sufficiently before entering phase III studies Treatment modalities, whose function and failure are well understood, appear most suitable for a rational design of combined therapeutic approaches needed to overcome the current limits of cancer therapy.
T. Wölfel, C. Huber, M ainz

\begin{tabular}{|c|c|}
\hline KARGER & (c) 2001 S. K arger G mbH , Freiburg \\
\hline $\begin{array}{l}\text { Fax +49 } 7614520714 \\
\text { E-mail Information@K arger.de }\end{array}$ & $\begin{array}{l}\text { A ccessible online at: } \\
\text { www.karger.com/journals/onk }\end{array}$ \\
\hline
\end{tabular}

\section{KARGER}

www.karger.com 